\title{
UWB Device for Breast Microwave Imaging: Phantom and Clinical Validations
}

\author{
Alessandro Vispa ${ }^{1}$, Lorenzo Sani ${ }^{1}$, Martina Paoli ${ }^{1}$, Alessandra Bigotti ${ }^{1}$, Giovanni Raspa ${ }^{1}$, Navid \\ Ghavami $^{1}$, Stefano Caschera ${ }^{1}$, Mohammad Ghavami ${ }^{2}$, Michele Duranti $^{3}$, Gianluigi Tiberi ${ }^{1,2}$ \\ ${ }^{1}$ UBT - Umbria Bioengineering Technologies, Spin off of University of Perugia, Perugia, Italy, \\ ${ }^{2}$ School of Engineering, London South Bank University, London, UK, \\ ${ }^{3}$ Department of Diagnostic Imaging, Perugia Hospital, Perugia, Italy.
}

Abbreviated title: UWB Device for Breast Microwave Imaging

\section{Correspondence to:}

Gianluigi Tiberi, School of Engineering, London South Bank University, London, UK

E-mail: g.tiberi@ing.unipi.it, Phone: +39 3490564302

Declaration of interest: Alessandro Vispa, Lorenzo Sani, Martina Paoli, Alessandra Bigotti,

Giovanni Raspa, Stefano Caschera are employed by UBT - Umbria Bioengineering Technologies.

Lorenzo Sani, Navid Ghavami and Gianluigi Tiberi are shareholders of UBT - Umbria Bioengineering Technologies. 


\begin{abstract}
Microwave imaging has received increasing attention in the last decades, motivated by its application in diagnostic imaging. Such effort has been encouraged by the fact that, at microwave frequencies, it is possible to distinguish between tissues with different dielectric properties. In such framework, a novel microwave device is presented here. The apparatus, consisting of two antennas operating in air, is completely safe and non-invasive since it does not emit any ionizing radiation and it can be used for breast lesion detection without requiring any breast crushing. We use Huygens Principle to provide a novel understanding into microwave imaging; specifically, the algorithm based on this principle provides images which represent homogeneity maps of the dielectric properties (dielectric constant and/or conductivity). The experimental results on phantoms having inclusions with different dielectric constants are presented here. In addition, the capability of the device to detect breast lesions has been verified through clinical examinations on 51 breasts. We introduce a metric to measure the non-homogenous behaviour of the image, establishing a modality to detect the presence of inclusions inside phantoms and, similarly, the presence of a lesion inside a breast.
\end{abstract}

Keywords: ultra wideband technology, microwave imaging, breast imaging, dielectric properties. 


\section{Introduction}

Several unique properties of ultra wideband (UWB), including its non-ionizing signals, low cost and the ability to penetrate through media (air, skin, bones and tissues) have transformed it into an ideal candidate to be used as a novel medical imaging technology.

Current medical imaging technologies are able to produce reconstructions of living tissues using a range of methods. Most common examples are ultrasound scanners, planar X-ray, X-rays computed tomography (CT) and nuclear magnetic resonance imaging (known as MRI). Each one of these techniques has its own drawbacks; for instance, ultrasound is a very cost effective technique and is extremely useful for selected tissues, but suffers from contrast problems and an inability to image objects with large acoustic impedance differences, e.g. air spaces and bones [1]. CT on the other hand, is very effective for imaging high contrast subjects but does result in a significant dose of ionizing radiation to the patient [2]. MRI is non-ionizing, offers excellent soft tissue contrast imaging, but scanners are expensive to buy and maintain. In response to all these limitations, UWB imaging, based on purely electrical field at microwave frequencies, has been recognized as a promising non-ionizing and non-invasive alternative imaging technology. With UWB imaging, tissues are pictured based on differences in their dielectric properties [3].

UWB microwave imaging has attracted growing attention, especially for its applicability to breast cancer detection [4], motivated by the significant contrast in the dielectric properties at microwave frequencies of normal and malignant tissues. UWB microwave imaging introduces some advantages with respect to the conventional examinations. First of all, UWB microwave imaging is free from ionizing radiations (X-rays), which are not recommended for screening purposes in women under the age of $50[5,6]$. Moreover, during the examinations carried out with UWB microwave imaging, the patient appears to be more comfortable since this type of measurement does not require the painful compression of the breast. The physical principle of the UWB microwave imaging techniques lies in a different perspective of the breast; in fact, rather than the density, microwaves respond differently if they hit tissues having different dielectric properties. A significant contrast between healthy breast tissue and malignant tissue is present at microwave frequencies; this contrast is shown to be up to a factor of 5 in conductivity and permittivity [4]. Meanwhile, newer studies suggest the existence of this contrast only between fatty and malignant breast tissues, and a lower contrast (as low as $10 \%$ in dielectric properties) between healthy fibro glandular and malignant tissues $[7,8]$. 
Current ongoing research on microwave breast imaging is separated mainly into microwave tomography and UWB radar techniques. Microwave tomography attempts to reconstruct the full electrical profile of the breast by solving a rather ill-posed and nonlinear inverse scattering problem $[9,10]$. Radar-based imaging requires solving a simpler problem of finding the microwave scattering map based on the differences in dielectric properties of the breast tissues [11-13]. Low signal to clutter (S/C) ratio and, in microwave tomography, complex mathematical formulation causing solution instability are some of the drawbacks of such microwave techniques.

At present, some UWB microwave imaging prototypes for breast cancer detection are at clinical trials stage $[14,15]$. Recently, a novel microwave apparatus (MammoWave, UBT Srl, Perugia, IT) started clinical validation [16, 17]. In MammoWave, microwave signals are processed through Huygens principle (HP) [18]. The methodology allows to differentiate between different tissues, or different conditions of tissues, and to get a final image, which represents a homogeneity map of the dielectric properties (dielectric constant and/or conductivity). UWB microwave imaging technology permits the use of all the information in the frequency domain by combining the information of the individual frequencies to build such image. Like radar-based imaging, HP method can identify the presence and the position of significant dispersions, i.e. inclusions within a volume. In [19], it has been shown that HP has superior performances in terms of $\mathrm{S} / \mathrm{C}$ ratio and in localization.

In this paper, we present a brief description of the MammoWave and the results achieved through phantom validations. Specifically, both homogenous phantoms and phantoms having inclusions are investigated. We introduce a metric to measure the non-homogenous behaviour of the microwave image, which is the ratio of maximum to the average of the image intensity (MAX/AVG), establishing a simple and effective modality to detect the presence of inclusions inside phantoms. In addition, the capability of the device to detect breast lesions has been verified through clinical examination on 51 breasts, each one with the correspondent output of the radiologist study review obtained using echography and/or mammography and/or MRI. Welch's t-test has been used to verify if MAX/AVG of the healthy breasts is statistically different from MAX/AVG of the non-healthy breasts; next, MAX/AVG has been successfully used for discriminating between healthy breasts and breast with lesions, with a true positive rate of 0.7 and a corresponding false negative rate of 0.35 . 
The rest of this manuscript is organized as follows. Section 2 describes the device and the fundamentals of the mathematical method used in the imaging procedure. Section 3 explains the metric to measure the nonhomogenous behaviour. In Section 4, we will show the results obtained, and Sections 5 and 6 are presenting some discussions and conclusions, respectively.

\section{Materials and Methods}

In accordance with the HP, 'Each locus of a wave excites the local matter which reradiates a secondary wavelet, and all wavelets superpose to a new, resulting wave (the envelope of those wavelets), and so on' [20]. Specifically, we consider what happens if we apply HP to reconstruct the field inside an object using the field measured outside it as the locus of a wave.

In more details, let us refer to the problem depicted in Fig. 1. A cylindrical phantom is illuminated by a transmitting antenna $\mathrm{tx}_{m, p}$ that operates in a given frequency band $\mathrm{B}$. The phantom has dielectric constant $\varepsilon_{r}$ and the conductivity $\sigma$ (which may be unknown). The phantom may contain an inclusion with a different dielectric constant and/or conductivity. The problem consists of identifying the presence of the inclusion by using the field measured through a receiving antenna which collects the measurements at many points $\mathrm{rx}_{n}$ displaced all around the phantom. As described in [21], it has been shown through both simulation and measurement in an anechoic chamber that the reconstructed field in the object will exhibit a mismatch in the region of transition of the two media, i.e. where the inclusion is located. This mismatch allows detection.

Instead, all images and results shown in this paper are obtained using the device MammoWave installed at the Department of Diagnostic Imaging, Perugia Hospital, Perugia, Italy.

The device, shown in Fig. 2, consists of two antennas operating in air, in the frequency band B from 1 to 9 GHz. The antennas are connected to a Vector Network Analyzer (Cobalt C1209, Copper Mountain, Indianapolis, IN). The device's internal surfaces are shielded with absorbing material (flat absorber $\mathrm{AH}$ model, SIEPEL Cyber, France). The instrument (appropriately integrated with a bed) is additionally constituted by: a hub with a cup that is placed to contain the breast of the patient (prone positioned) or, as in this case, the phantoms; two arms to associate transmitter (Tx) and receiver (Rx) to the hub through rotation. 
The Tx antenna is a horn antenna having a Voltage Standing Wave Ratio (VSWR) lower than 3.0 between 1 and $1.6 \mathrm{GHz}$ and lower that 2.0 between 1.6 and $9 \mathrm{GHz}$. The Rx antenna is a microstrip monopole antenna having VSWR lower than 2.0 between 1 and $9 \mathrm{GHz}$. The Tx and Rx antennas are placed at the same height and can be circularly moved on the azimuthal plane, to irradiate (Tx) and subsequently receive ( $\mathrm{Rx})$ the microwave signals from different angular directions. The Tx plane is divided into 5 angular sections of $72^{\circ}$. For each section, the transmitting antenna can transmit from the central position of the section $\left(0^{\circ}, 72^{\circ}, 144^{\circ}\right.$, $216^{\circ}, 288^{\circ}$ ) plus from two other positions displaced $\pm 4.5^{\circ}$ from the central one. It follows that we will have a triplet for each section. For each Tx position, the Rx antenna can be moved measuring the received signal every $4.5^{\circ}$; this will lead to a total of 80 receiving points.

In more details, assuming that $\mathrm{Rx}$ can be rotatably moved to measure the received signal at the points $\mathrm{rx}_{n} \equiv\left(a_{0}, \phi_{n}\right) \equiv \vec{\rho}_{n}$ displaced along a circular surface having radius $a_{0}$ as shown in Fig. 1, the received signals can be expressed as:

$$
S 21_{n}^{m, p}\left(a_{0}, \phi_{n} ; \mathrm{tx}_{m, p} ; f\right)
$$

where $n=1,2, \ldots, 80$, indicates the receiving points; $m=1,2 \ldots, 5$ indicates the transmitting sections, $p=1,2,3$ indicates the position inside each transmitting section; and $f$ is the frequency. The received signals are then processed through HP to calculate the field inside the cylinder; such field is then used to generate an image, which is a homogeneity map of dielectric properties. Specifically, we calculate the field inside the cylinder as a superposition of the fields radiated by the $N_{P T}$ receiving points of eq. (1):

$$
E_{H P, 2 D}^{r c s t r}\left(\rho, \phi ; \mathrm{tx}_{m, p} ; f\right)=\sum_{n=1}^{N_{P T}} S 21_{n}^{m, p}\left(a_{0}, \phi_{n} ; \mathrm{tx}_{m, p} ; f\right) G\left(k_{1}\left|\vec{\rho}_{n}-\vec{\rho}\right|\right)
$$

where $(\rho, \phi) \equiv \vec{\rho}$ is the observation point, $k_{1}$ here represents the wave number. Due to the presence of both Tx and Rx antennas in air, the wave number $k_{1}$ is set using free space dielectric constant. In eq. (2), the string rcstr is used to indicate the "reconstructed" internal field, while the string HP indicates that a HP based procedure will be employed. In eq. (2), the Green's function $G$ for homogenous media is used to propagate the field. It should be highlighted that eq. (2) does not give the correct internal field, even when dealing with homogenous problems. However, it has been shown in $[18,21]$ that eq. (2) can capture the contrast, i.e. mismatch boundaries. Assuming we use $N_{F}$ frequencies $f_{i}$ in the band $\mathrm{B}$, it follows that the intensity of the image I may be obtained through the following equation, i.e. by summing incoherently all the solutions: 


$$
I\left(\rho, \phi ; \mathrm{tx}_{m, p}\right)=\sum_{i=1}^{N_{F}}\left|E_{H P}^{r c s t r}\left(\rho, \phi ; \mathrm{tx}_{m, p} ; f_{i}\right)\right|^{2}
$$

To remove the artefacts [18], here we employ the subtraction between S21 obtained using two measurements belonging to the triplet of the same section. In formula:

$$
E_{H P, 2 D}^{r c s t r}\left(\rho, \phi ; \mathrm{tx}_{m, p}-\mathrm{tx}_{m, p^{\prime}} ; f\right)=\sum_{n=1}^{N_{p r}}\left(S 21_{n}^{m, p}\left(a_{0}, \phi_{n} ; \mathrm{tx}_{m, p} ; f\right)-S 21_{n}^{m, p^{\prime}}\left(a_{0}, \phi_{n} ; \mathrm{tx}_{m, p^{\prime}} ; f\right)\right) G\left(k_{1}\left|\vec{\rho}_{n}-\vec{\rho}\right|\right)
$$

The images of all the sections are then summed up to get a final image as following:

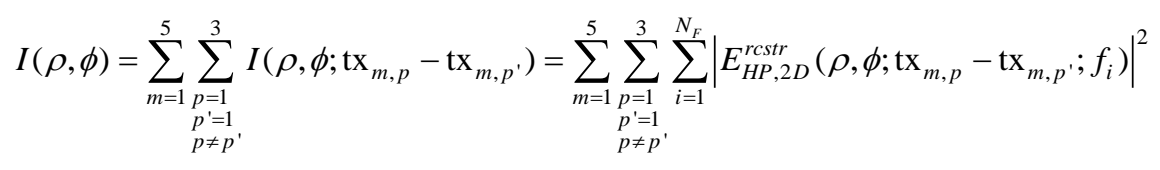

The capability of the device to detect inclusions has been verified through measurements in phantoms. In this context, a $1 \mathrm{~mm}$ thick plastic cylinder with $11 \mathrm{~cm}$ diameter and $23 \mathrm{~cm}$ height has been used as a container. The samples taken into examination have been oil (in more details, sunflower oil) and glycol (in more details, a solution constituted of $85 \%$ glycol and $15 \%$ water). To reproduce the inclusions, tubes of $10 \mathrm{~mm}$ diameter and $23 \mathrm{~cm}$ height filled with water or glycol have been used. The following measurements have been carried out:

i) Homogeneous phantom consisting of oil,

ii) Homogeneous phantom consisting of glycol,

iii) Phantom consisting of oil with an inclusion consisting of glycol,

iv) Phantom consisting of oil with an inclusion consisting of water,

v) Phantom consisting of glycol with an inclusion consisting of water.

Next, the capability of the device to detect breast lesions has been verified through clinical trials which are ongoing under a protocol approved by the Ethical Committee of Regione Umbria, Italy (N. 6845/15/AV/DM of 14/10/2015, N. 10352/17/NCAV of 16/03/2017). The protocol concerns a feasibility study for detection of breast lesions using the proposed microwave mammogram apparatus. In this context, we present the results obtained using 51 breasts, each one with the correspondent output of the radiologist study review obtained using echography and/or mammography and/or MRI. Echography was performed using the MyLab 70 xvg Ultrasound Scanner (Esaote, Genova, Italy); mammography was performed using Selenia LORAD Mammography System (Hologic, Marlborough, MA); magnetic resonance imaging was performed through a 3T scanner (Siemens Healthcare, Erlangen, Germany). The output of the radiologist study review has been 
used as gold standard for classification of the breasts in two categories: healthy (i.e. breasts with no lesion) and non-healthy (i.e. breasts containing lesions).

The informed consent was obtained from all the patients. All procedures performed in this study were in accordance with the ethical standards of the institutional and/or national research committee and with the 1964 Helsinki declaration and its later amendments or comparable ethical standards.

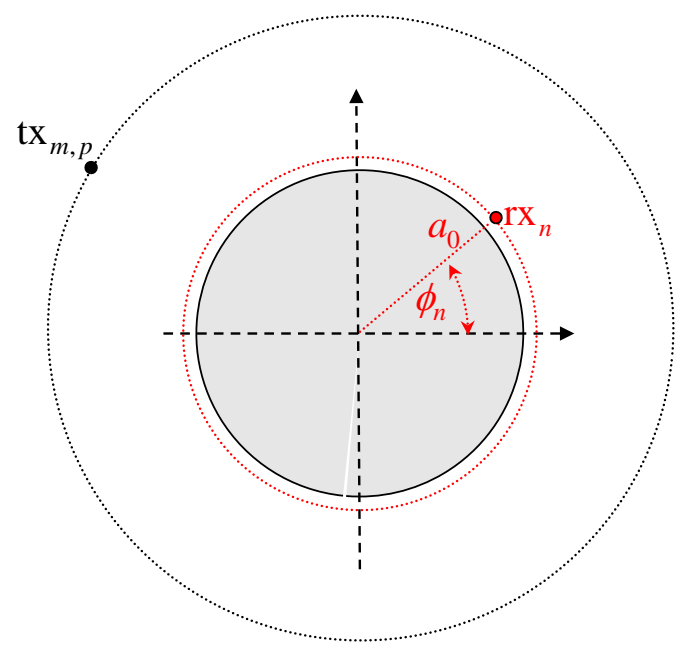

Fig. 1: Pictorial view of the problem, where the cylindrical phantom/breast is shown in light gray, and the red and black dots represent the receiving and transmitting antennas respectively (color online). The red and black dotted lines represent the circumferences where the receiving and transmitting antennas may be circularly moved on the azimuthal plane to irradiate and subsequently receive the signals from different directions. 

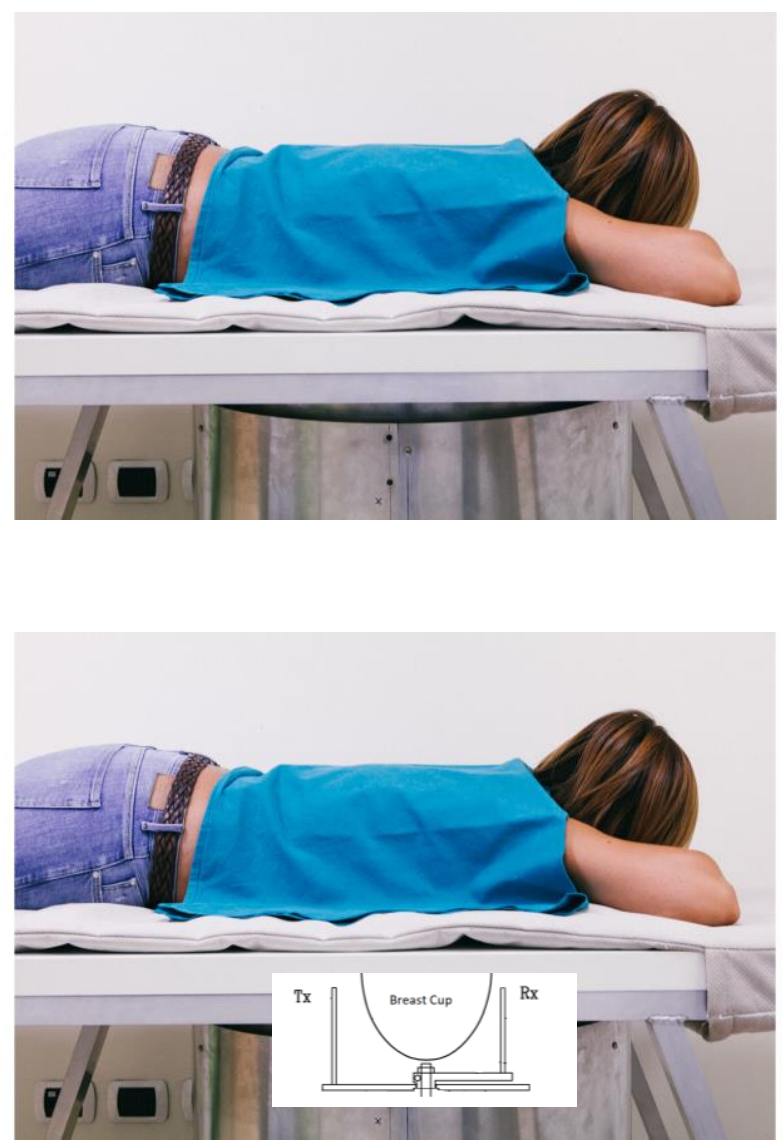

Fig. 2: Top) MammoWave device installed at the Department of Diagnostic Imaging, Perugia Hospital, Perugia, Italy; bottom) The device includes a cup that holds the breast when the patient lies prone on the examination table. The transmitting (Tx) and receiving $(\mathrm{Rx})$ antennas are located inside a hub and can be moved around the azimuth, to irradiate the breast (through Tx) and capture the microwaves scattered by the breast itself (through Rx). Both Tx and Rx are connected to a Vector Network Analyzer (VNA). No matching liquid is required.

\section{Calculations}

For each measurement, considering the 15 positions of $\mathrm{Tx}$, the 80 receiving positions of $\mathrm{Rx}$ and the frequencies in the range $1-9 \mathrm{GHz}$ with a $5 \mathrm{MHz}$ step, we generate a $1200 \times 1601$ raw data matrix of $S_{21}$ complex values, with a measurement time of approximately 10 minutes. For each measurement, the radius $a_{0}$ is $0.07 \mathrm{~m}$. Correspondent microwave images have been obtained by employing eq. (5), with a computational time of approximately 5 minutes.

In order to compare different measurements, all images have been normalized to their own average intensity. In addition, we introduce a metric to measure the non-homogenous behavior of the image, which is the ratio between maximum and average of the image intensity (MAX/AVG). In order to assess the repeatability, 
imaging of homogeneous phantoms has been performed five times, and the mean and standard deviation of MAX/AVG have been determined.

With the aim of investigating the impact of different sub-bands, four images of oil with glycol inclusion are generated employing eq. (5) for the four $2 \mathrm{GHz}$ sub-bands (i.e. $1-3 \mathrm{GHz}, 3-5 \mathrm{GHz}, 5-7 \mathrm{GHz}$, and 7-9GHz) separately.

To be able to perform intra-breasts comparison, all images have been normalized to unitary average. For each microwave image, we calculated the parameter MAX/AVG. In addition, we also calculated the coefficient of variation $(\mathrm{CV})$, i.e. the ratio of the standard deviation to the mean of the images, which is a metric conventional used to measure the non-homogenous behavior of images. Based on the classification performed by the radiologist, Welch's t-test (i.e. a two-sample two-tailed unpooled variances t-test) with $\alpha=$ 0.05 has been performed for MAX/AVG. The same t-test procedure has been repeated for CV.

Next we calculated: the mean and standard deviation of MAX/AVG and of CV for the healthy breasts; the mean and standard deviation of MAX/AVG and of CV for the non-healthy breasts.

Finally, we empirically evaluated the receiver operating characteristic (ROC) curve using, separately, MAX/AVG or CV for classification. Specifically, since True Positive (TP) rate and False Negative (FN) rate depend on the classifier threshold, i.e. the decision offset, we empirically calculated ROC curves by adjusting the decision offset and calculating TP and FN for all possible decision offsets. It is worthwhile pointing out that TP rate is commonly denoted as sensitivity, while FN rate is equal to 1-specificity.

\begin{tabular}{|c|c|c|}
\hline & Dielectric constant (at 5 GHz) & Conductivity (at 5 GHz) [S/m] \\
\hline Oil (sunflower) & 4 & 1 \\
\hline $\begin{array}{c}\text { Glycol (a solution constituted of } \\
\mathbf{8 5 \%} \text { glycol and 15\% water) }\end{array}$ & 15 & 3 \\
\hline Water & 77 & 0 \\
\hline
\end{tabular}

Table 1. Dielectric properties at $5 \mathrm{GHz}$ 


\section{Results}

\section{1. Phantom Imaging}

The dielectric properties of oil, glycol, and water for the frequency equal to $5 \mathrm{GHz}$ are given in Table 1 . The dielectric property of oil was measured using the Epsilon dielectric measurement device (Biox Epsilon Model E100 manufactured by Biox System Ltd Company), which is an instrument for contact measurement using a fingerprint sensor that responds to capacitance. It can be used for measurements on isolated soft solids and liquids. It is important to highlight that the measurement device does not give the dielectric value at the frequency of the interest but gives the permittivity values at frequency of $0 \mathrm{~Hz}$. However, it is still suitable for a liquid which consists of ingredients that do not experience any changes with frequency variation such as oil. The conductivity of the oil can be neglected [22], while the dielectric properties of glycol and water were derived from [23].

To assess the repeatability, imaging of homogeneous phantoms has been performed five times: concerning the homogeneous phantom of oil, the mean MAX/AVG of the five imaging experiment is equal to 1.42 , with a standard deviation of 0.015 . Regarding the homogeneous phantom of glycol, the mean MAX/AVG is equal to 1.58 , with a standard deviation of 0.02 .

An image of the homogeneous phantom of oil is given in Fig 3. Fig. 4a shows the images of the oil with glycol inclusion having $10 \mathrm{~mm}$ diameter, and Fig. 4b refers to the oil with water inclusion having $10 \mathrm{~mm}$ diameter. Fig. $4 \mathrm{c}$ refers to the glycol with water inclusion.

Fig. 5 shows the images of oil with glycol inclusion obtained employing eq. (5) for the four $2 \mathrm{GHz}$ subbands, separately. This allows investigating the impact of different sub-bands in the detection process.

Table 2 shows the MAX/AVG for the oil with glycol inclusion, for oil with water inclusion and glycol with water inclusion calculated when employing the full band (1- $9 \mathrm{GHz})$ and when employing the four different sub-bands, separately.

\section{2. Clinical Imaging}

According to the radiologist study review, 22 healthy breasts (16 dense) and 29 non-healthy breasts (21 dense) underwent microwave imaging (which included 7 carcinoma, 9 fibroadenoma, and 5 microcalcifications). More details on the patient population are given in Table 3. Table 4 summarizes the 
mean and standard deviation of MAX/AVG and of CV for the healthy breasts and for the non-healthy breasts.

As an example, in the left column of Fig. 6, microwave images are given for: (a) heterogeneously dense breast with carcinoma; (b) scattered fibro glandular healthy breast; (c) heterogeneously dense breast with microcalcifications; (d) heterogeneously dense breast with fibroadenoma. The tables, shown as inserts, summarize the correspondent MAX/AVG and CV. In the right column of Fig. 6, the images obtained using mammography (a, b, c) or echography (d) are given.

Fig 7 shows the box plot of the Welch's t-test $(\alpha=0.05)$ performed for MAX/AVG and for CV, while ROC curves are given in Fig 8.

\begin{tabular}{|l|c|c|c|c|c|}
\hline & $\begin{array}{c}\mathbf{1 - 9} \\
\mathbf{G H z}\end{array}$ & $\begin{array}{c}\mathbf{1 - 3} \\
\mathbf{G H z}\end{array}$ & $\begin{array}{c}\mathbf{3 - 5} \\
\mathbf{G H z}\end{array}$ & $\begin{array}{c}\mathbf{5 - 7} \\
\mathbf{G H z}\end{array}$ & $\begin{array}{c}\mathbf{7 - 9} \\
\mathbf{G H z}\end{array}$ \\
\hline Oil with glycol inclusion & 1.95 & 1.80 & 1.82 & 2.05 & 2.36 \\
\hline Oil with water inclusion & 2.40 & 2.28 & 2.46 & 2.50 & 2.88 \\
\hline Glycol with water inclusion & 1.87 & 1.85 & 2.11 & 2.00 & 1.81 \\
\hline
\end{tabular}

Table 2. MAX/AVG for the oil with glycol inclusion, for oil with water inclusion and glycol with water inclusion calculated when employing the full band and when employing the four different sub-bands, separately.

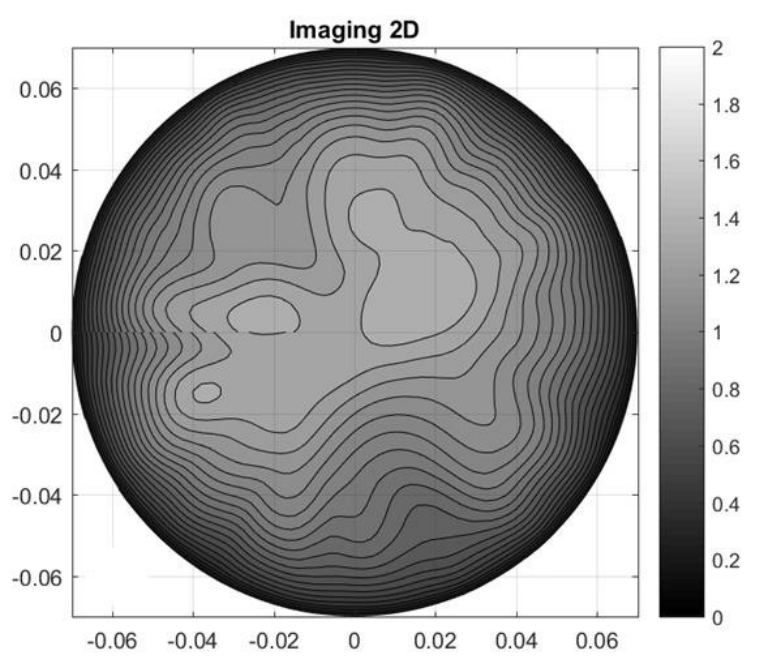

Fig. 3. Image of the homogeneous phantom of oil. The image has been normalized to its own average intensity. $\mathrm{X}$ and $\mathrm{Y}$ are given in meters, while the intensity is in arbitrary unit. 


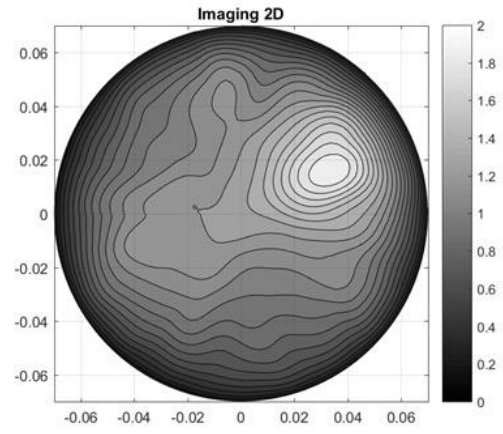

(a)

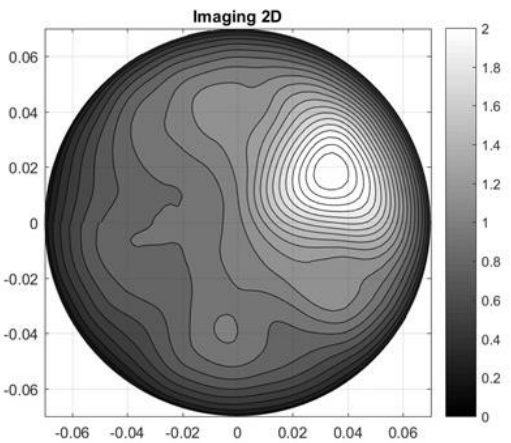

(b)

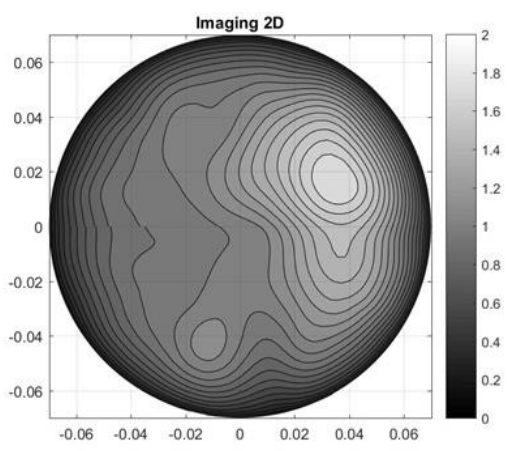

(c)

Fig. 4. (a) Image of the oil with glycol inclusion; (b) image of the oil with water inclusion; (c) image of the glycol with water inclusion. The images have been normalized to their own average intensity. $\mathrm{X}$ and $\mathrm{Y}$ are given in meters, while the intensity is in arbitrary unit.

\section{Discussion}

Healthy breast tissues may vary upon the percentages of adipose, i.e. fatty, and fibro glandular tissues [7, 8]. In this context, the dielectric properties of the oil used in this study may be considered a good approximation of the dielectric properties of high adipose-content healthy breast tissue, while the dielectric properties of the glycol may be considered a good approximation of the dielectric properties of low adipose-content healthy breast tissue. The dielectric properties of the water may be considered as an approximation of the malignant tissue.

Images obtained using the proposed apparatus are intensity maps representing the homogeneity of dielectric properties. However, as highlighted in Fig 3, even homogenous phantom will lead to a slight nonhomogeneous map. This is due to the fact that the antennas operate in the air, i.e. there is a mismatch between the medium where the antennas operate, and the phantom. In addition, the image in Fig 3 does not show symmetry. As described before, all the images are obtained by combining results of the 5 sections, which are derived using the correspondent 5 triplets of $S_{21}$ values. Slight differences in the 5 triplets $(<0.1 \%)$, due to the intrinsic stability of the VNA and the tolerance in mechanical manufacturing of the device are presumably the reason of such asymmetry. In this context, it should be pointed out that particular care has been taken to guarantee stability of the coaxial connections in the apparatus, thus adopting the use of semirigid coaxial cabling. 
The non-homogeneous behavior can be quantified by introducing a dedicated metric, which is MAX/AVG. Specifically, we calculated that MAX/AVG is equal to 1.42 for the homogeneous phantom of oil, and equal to 1.58 for the homogeneous phantom of glycol. The higher value of MAX/AVG for the homogeneous phantom of glycol with respect to the homogeneous phantom of oil is probably due to the higher dielectric constant of the glycol.

Let us now consider Fig. 4: the contrast due to the differences in dielectric properties is highlighted by an increase in the intensity in the region where the inclusion is placed. Thus, if inside a phantom there is an inclusion, i.e. a mismatch in dielectric properties exists, such inclusion will lead to a peak in intensity.

Consequently, MAX/AVG for the phantom with inclusion will be greater than MAX/AVG for the homogenous phantom. Specifically, concerning the oil, we found that MAX/AVG is equal to 1.95 when the inclusion is constituted of glycol (which is $37 \%$ higher that MAX/AVG calculated in the homogeneous phantom), and it increases up to 2.40 if the inclusion in constituted of water (which is $69 \%$ higher that MAX/AVG calculated in the homogeneous phantom). Such enhancement may be related to the higher contrast in dielectric properties between oil and water.

Concerning the glycol, we found that MAX/AVG is equal to 1.87 when the inclusion is constituted of water (which is $18 \%$ higher that MAX/AVG calculated in the homogeneous phantom). This lower value can be explained by recalling that glycol has much higher losses than oil, and losses may compromise the penetration of the field.
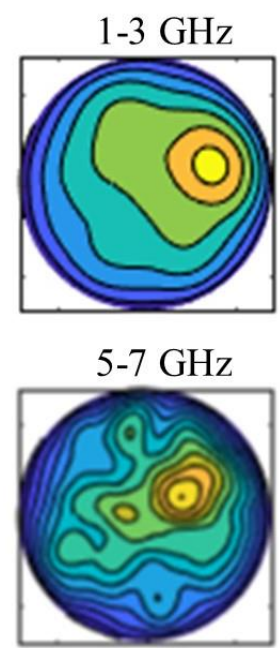

$3-5 \mathrm{GHz}$

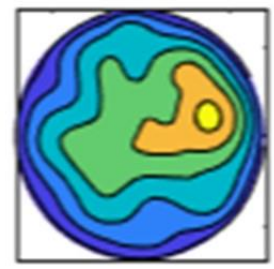

7-9 GHz

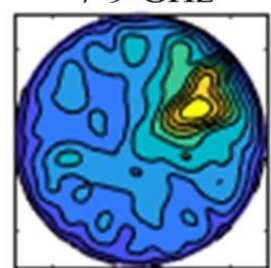

Fig. 5. Images of oil with glycol inclusion obtained employing eq. (5) for the four $2 \mathrm{GHz}$ sub-bands, separately. All the images are normalized to the correspondent maximum. 
As shown in Fig. 5, which refers to the oil with glycol inclusion, the presence and location of the inclusion is visible for all sub-bands, although the characteristics of the peak varies with the frequency itself. Specifically, it can be noted that the dimension of the peak decreases with the frequency. This confirms the findings presented in [21], where it is concluded that higher frequency leads to an increase of resolution.

Table 2 shows MAX/AVG for oil with glycol inclusion, for oil with water inclusion and for glycol with water inclusion calculated in four different sub-bands. Interestingly, it can be noted that, for the oil with glycol inclusion and for the oil with water inclusion, MAX/AVG increases with the frequency. This trend is also confirmed in Fig 5, since a better definition of the peak at higher frequencies implies a decreasing of the average value in the intensity of the image.

Conversely, for glycol with water inclusion, MAX/AVG first increases for the first sub-bands and then it has a drop. Again, this can be explained recalling that glycol has higher losses than oil: losses compromise the penetration of the field, especially at higher frequencies. As the breast is constituted of lossy tissues $[4,7,8]$, we decided to perform the clinical imaging employing the full band.

Concerning clinical images, images obtained using the proposed apparatus are intensity maps representing the homogeneity of tissues' dielectric properties. Microwave images are given here as 2D images in the azimuthal plane, i.e. coronal plane. As the breast is constituted of non-homogeneous tissues $[4,7,8]$, a certain level of non-homogeneity can also be seen in the healthy breast images, as highlighted in Fig 6b (left); such non-homogeneity will be related to the subjects' breast anatomy. However, non-homogeneity is more pronounced in non-healthy breasts, as highlighted in Figs 6a (left), 6c (left) and 6d (left). From Table 4, it is possible to conclude that microwave images of non-healthy breasts have a mean MAX/AVG of approximately $7 \%$ greater than those of the healthy breasts, and that microwave images of non-healthy breasts have a mean CV of approximately 3\% greater than those of the healthy breasts. Welch's t-test $(\alpha=$ 0.05) for MAX/AVG leads to a P value of 0.0065 , while the same t-test for CV leads to a P value of 0.0765 . Thus, it follows that the metric MAX/AVG is statistically more robust than CV for discriminating between healthy breasts and breasts with lesions; this is also confirmed by comparing the two the ROC curves shown in Fig 8. From MAX/AVG ROC curve, we obtained $\mathrm{TP}=0.7$ with a corresponding $\mathrm{FN}=0.35$, values which are not-inferior to [24] where, although, performances have been statistically evaluated using clinical healthy breasts data and injecting simulated tumor response to mimic non-healthy breasts data. 


\begin{tabular}{|l|c|c|c|c|c|c|c|}
\hline & $\begin{array}{l}\text { Total } \\
\text { cases (n) }\end{array}$ & $\begin{array}{l}\text { Mean age } \\
(\mathbf{y})\end{array}$ & $\begin{array}{l}\text { Age } \\
\text { range (y) }\end{array}$ & Carcinoma & Fibroadenoma & Microcalcifications & $\begin{array}{l}\text { Other } \\
\text { lesions }\end{array}$ \\
\hline $\begin{array}{l}\text { Healthy } \\
\text { breasts }\end{array}$ & 22 & 45 & $29-70$ & - & - & - & - \\
\hline $\begin{array}{l}\text { Non- } \\
\text { healthy } \\
\text { breasts }\end{array}$ & 29 & 52 & $20-86$ & 7 & 9 & 5 & 8 \\
\hline
\end{tabular}

Table 3. Patient population.

\begin{tabular}{|c|c|c|c|c|}
\hline & $\begin{array}{c}\text { mean of MAX/AVG of } \\
\text { the microwave images }\end{array}$ & $\begin{array}{c}\text { standard deviation of } \\
\text { MAX/AVG of the } \\
\text { microwave images }\end{array}$ & $\begin{array}{c}\text { mean of CV of the } \\
\text { microwave images }\end{array}$ & $\begin{array}{c}\text { standard deviation } \\
\text { of CV of the } \\
\text { microwave images }\end{array}$ \\
\hline healthy breasts & 1.65 & 0.08 & 0.417 & 0.013 \\
\hline non-healthy & 1.76 & 0.11 & 0.426 & 0.016 \\
\hline
\end{tabular}

Table 4- Mean and standard deviation of MAX/AVG and of CV for healthy and non-healthy breasts, separately. 


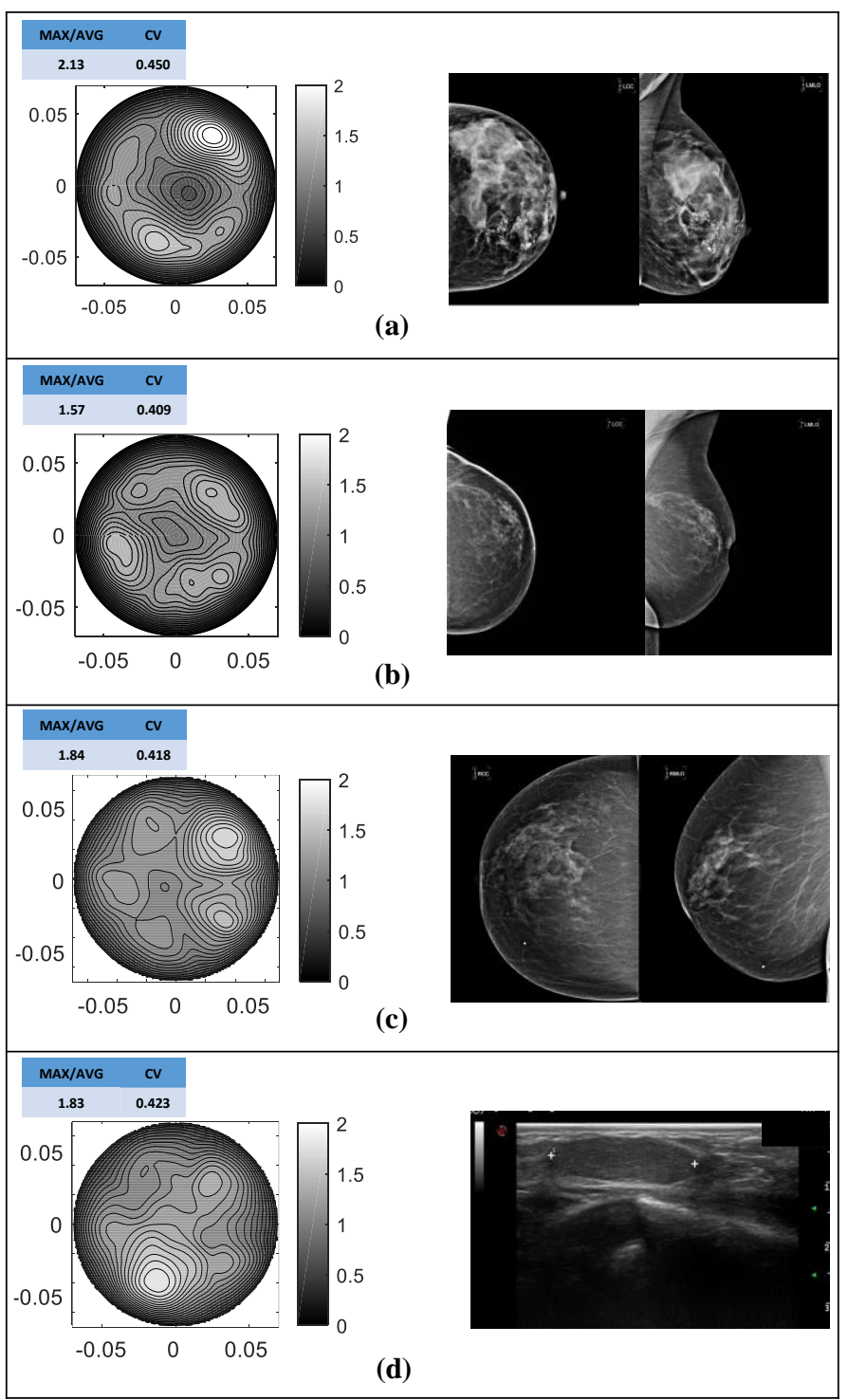

Fig. 6. Left column: microwave images are given for (a) heterogeneously dense breast with carcinoma; (b) scattered fibro glandular healthy breast; (c) heterogeneously dense breast with microcalcifications; (d) heterogeneously dense breast with fibroadenoma. The images have been normalized to their own average intensity. $\mathrm{X}$ and $\mathrm{Y}$ are given in meters, while the intensity is in arbitrary unit. Right column: correspondent images obtained using mammography (a, b, c) or echography (d). 

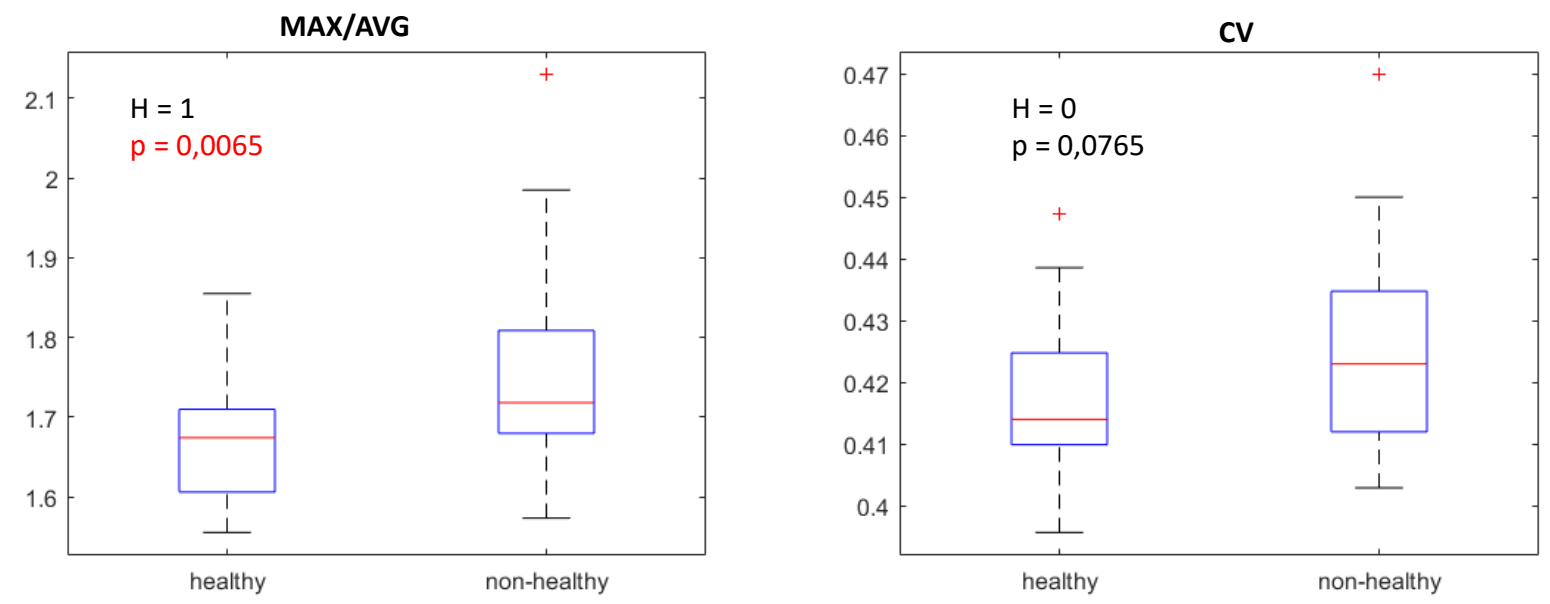

Fig. 7. Boxplots of the Welch's t-test $(\alpha=0.05)$ performed for MAX/AVG (left) and for CV (right). In the Boxplots, the central mark is the median, the edges of the box are the 25 th and 75 th percentiles, the whiskers extend to the most extreme data points the algorithm considers to be not outliers, and the outliers are plotted individually.

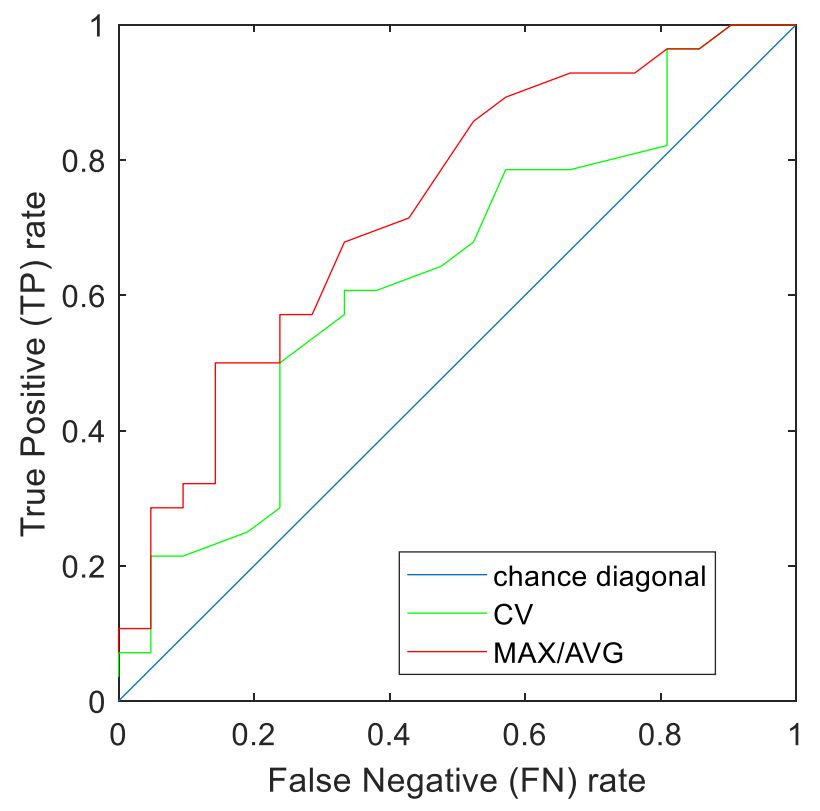

Fig. 8. Receiver operating characteristic (ROC) curves obtained using, separately, MAX/AVG or CV for classification. We empirically calculated ROC curves by adjusting the decision offset and calculating TP and FN for all possible decision offsets. 


\section{Conclusions}

A new microwave imaging device based on the Huygens principle has been validated through phantom measurements. The device allows achieving all the information in the frequency domain to be used by combining the single frequency information to construct an image. The procedure is robust and permits, in the presence of a contrast in dielectric properties, the detection of inclusions within an object. Validation of the device through experiments has been performed and presented, illustrating its effectiveness. It has been shown that MAX/AVG of phantoms with inclusion is up to $69 \%$ greater than MAX/AVG of phantoms without inclusion.

The introduction of a metric to measure the non-homogenous behaviour of the image, which is the ratio of maximum to the average of the image intensity (MAX/AVG), is fundamental to quantify the detection rate. Specifically, the capability of the device to detect breast lesions has been verified through clinical trials. It has been shown that microwave images of non-healthy breasts have a mean MAX/AVG of approximately 7\% greater than those of the healthy breasts. Welch's t-test for MAX/AVG of the healthy breasts and MAX/AVG of the non-healthy breasts leads to a $\mathrm{P}$ value of 0.0065 ; by conventional criteria, this difference is considered statistically significant. It follows that MAX/AVG of microwave images could be used for discriminating between healthy breasts and breast with lesions, with a true positive rate of 0.7 and a corresponding false negative rate of 0.35 . As there is no ionising radiation, the device is safe to use at any age, in any condition, with no limit on frequency of breast screenings. Moreover, in contrast to traditional mammography units that compress the breast, the device is more comfortable for the patient. The device does not use matching liquid, i.e. the antennas operate in the air; this makes the device manufacturing and functioning simpler. The absence of matching liquid enhances unwanted reflections from the breast surface; however, such reflections are successfully removed through artefacts removal procedure.

Research is in progress to find the metric, which maximizes detection rate and to evaluate how detection rate performances varies with respect to breasts parameters such as density and kind of lesions, including early stage cancers.

All the images shown here are non-filtered. Different kind of filters can be applied [25]; however, filtering highlights the peaks of intensities even if they do not correspond to any inclusions. Here instead, in order to 
compare images of homogeneous phantom and images of phantom with inclusions, and to perform intrabreasts comparison, all images have been normalized to their own average intensity.

Finally, it is worthwhile to point out that, together with detection, the device also allows localization. In this paper, localization has been only assessed visually. Errors in localization are expected due to the use of the wave number in free space [21] in eq. (2); a deeper investigation should be performed to analyze the error in localization, also with respect to the distance of the receiving points from the external surface of the object.

\section{Acknowledgements}

This project has received funding from the European Union's Horizon 2020 research and innovation programme under grant agreement No 830265.

This project leading to this application has received funding from the European Union's Horizon 2020 research and innovation programme under the Marie Sklodowska-Curie grant agreement No 793449. 


\section{References}

[1] C.R. Hill, G.R. Ter Harr, Review article: High intensity focused ultrasound-potential for cancer treatment, British Journal of Radiology 68 (816) (1995) 1296-1303.

[2] R.F. Cotellessa et.al Principle of Computerized Tomography, IEEE Free Press, (1987).

[3] S. Semenov, Microwave tomography: review of the progress towards clinical applications. Philosophical transactions Series A, Mathematical, physical, and engineering sciences, 367 (1900) (2009) 3021-3042. doi:10.1098/rsta.2009.0092.

[4] N.K. Nikolova, Microwave Imaging for Breast Cancer, IEEE Microwave Magazine 12 (7) (2011) 78-94.

[5] D.L. Miglioretti, J. Lange, J.J. van den Broek, C.I. Lee, N.T. van Ravesteyn, D. Ritley, et al, RadiationInduced Breast Cancer Incidence and Mortality From Digital Mammography Screening: A Modeling Study, Ann Intern Med., 164 (2016) 205-214.

[6] M.S. Fuller, C.I. Lee, J.G. Elmore, Breast Cancer Screening: An Evidence-Based Update, The Medical clinics of North America, 99 (3) (2015) 451-468.

[7] M. Lazebnik, L. McCartney, D. Popovic, C.B. Watkins, M.J. Lindstrom, J. Harter, S. Sewall, A. Magliocco, J.H. Booske, M. Okoniewski, et al, A large-scale study of the ultrawideband microwave dielectric properties of normal breast tissue obtained from reduction surgeries, Physics in medicine and biology 52 (10) (2007) 2637.

[8] M. Lazebnik, D. Popovic, L. McCartney, C.B. Watkins, M.J. Lindstrom, J. Harter, S. Sewall, T. Ogilvie, A. Magliocco, T.M. Breslin et al, A large-scale study of the ultrawideband microwave dielectric properties of normal, benign and malignant breast tissues obtained from cancer surgeries, Physics in Medicine and Biology 52 (20) (2007) 6093.

[9] P.M. Meaney, K.D. Paulsen, Nonactive antenna compensation for fixed-array microwave imaging: Part II imaging results, IEEE Trans. Med. Imag. 18 (6) 50851,8 (1999).

[10] D.W. Winters, B.D. Van Veen, S.C. Hagness, A sparsity regularization approach to the electromagnetic inverse scattering problem, IEEE Trans. Antennas Propag. 58 (1) (2010) 145-154.

[11] X. Li, E. J. Bond, B. D. V. Veen, and S. C. Hagness, An overview of ultra-wideband microwave imaging via space-time beamforming for early-stage breast-cancer detection, IEEE Antennas Propag. Mag., 47 (1) (2005) 19-34.

[12] F. Porter, Kirshin, E., Santorelli, A., Coates, M. \& Popović, M. Time-domain multistatic radar system for microwave breast screening. IEEE Antennas Wirel. Propag. Lett. 12 (2013) 229-232.

[13] A. Santorelli, et al. A time-domain microwave system for breast cancer detection using a flexible circuit board. IEEE Trans. Instrum. Meas. 64 (2015) 2986-2994.

[14] P.M. Meaney, M.W. Fanning, T. Raynolds, et al. Initial Clinical Experience with Microwave Breast Imaging in Women with Normal Mammography, Acad Radiol 14 (2) (2007) 207-218.

[15] D. Byrne, I.J. Craddock. Time-Domain Wideband Adaptive Beamforming for Radar Breast Imaging, IEEE Transactions on Antennas and Propagation 63 (4) (2015) 1725-1735.

[16] L. Sani, et al., Initial Clinical Validation of a Novel Microwave Apparatus for Testing Breast Integrity, IEEE International Conference on Imaging Systems and Techniques (IST) 278-282 (2016).

[17] G. Tiberi, R. Raspa. Apparatus for testing the integrity of mammary tissues. Patent n.0001413526, 2012.

[18] G. Tiberi, N. Ghavami, D.J. Edwards, A. Monorchio, Ultrawideband microwave imaging of cylindrical objects with inclusions, IET Microwaves, Antennas Propagation, 5 (12) (2011) 1440-1446. 
[19] N. Ghavami, P. Probert Smith, G. Tiberi, D. Edwards and I. Craddock, Non-iterative beamforming based on Huygens principle for multistatic ultrawide band radar: application to breast imaging, IET Microwaves, Antennas \& Propagation, 9 (12) (2015) 1233-1240.

[20] P. Enders, Huygens principle as universal model of propagation, Lat. Am. J. Phys. Educ., 3 (1) (2009) 19-31.

[21] N. Ghavami, G. Tiberi, D.J. Edwards, A. Monorchio, UWB Microwave Imaging of Objects With Canonical Shape, IEEE Transactions on Antennas and Propagation 60 (1) (2012) 231-239. doi: 10.1109/TAP.2011.2167905.

[22] J. Vrba, D. Vrba, Temperature and Frequency Dependent Empirical Models of Dielectric Properties of Sunflower and Olive Oil, Radioengineering, 22 (4) (2013) 1281-1287.

[23] P. M. Meaney, C. J. Fox, S. D. Geimer and K. D. Paulsen, Electrical Characterization of Glycerin: Water Mixtures: Implications for Use as a Coupling Medium in Microwave Tomography, IEEE Transactions on Microwave Theory and Techniques, 65 (5) (2017) 1471-1478.

[24] Y. Li, E. Porter, A. Santorelli, M. Popovi, M. Coates, "Microwave breast cancer detection via cost-sensitive ensemble classifiers: Phantom and patient investigation,” Biomedical Signal Processing and Control, 31 (2017) 366376.

[25] M. Z. Abdullah, S. A. Binajjaj, T. F. Zanoon, A. J. Peyton, High-resolution imaging of dielectric profiles by using a time-domain ultra wideband radar sensor, Measurement, 44 (5) (2011) 859-870. 